\title{
Nivel de conocimiento sobre prescripción antibiótica racional de los cirujanos dentistas, Chachapoyas, Perú, 2020
}

\section{Level of knowledge about rational antibiotic prescription of dental surgeons, Chachapoyas, Peru, 2020}

\author{
Katya Medina Terrones ${ }^{1}$, Franz Tito Coronel Zubiate ${ }^{2}$
}

\begin{abstract}
RESUMEN
El objetivo de la investigación fue determinar el nivel de conocimiento sobre prescripción antibiótica racional de los cirujanos dentistas en la ciudad de Chachapoyas en el año 2020. El presente estudio fue de enfoque cuantitativo. La muestra estuvo constituida por 30 cirujanos dentistas que cooperaron con la investigación. Para el registro de datos se aplicó la técnica: encuesta y como instrumento: cuestiona, los datos se analizaron con la estadística descriptiva de frecuencia. Los resultados evidencian que del 100\% de los encuestados el 70\% tuvo un conocimiento de nivel alto, el 16\% bajo y el 13.3\% alto. En la primera dimensión (PET) el 53\% tuvo un conocimiento de nivel medio, el $36.7 \%$ bajo y el 10\% alto. En la dimensión antibiótico de elección en pacientes alérgicos (AEPA) el 63.4\% tuvieron un conocimiento de nivel bajo,23.3\% medio y el 13.3\% alto. En la dimensión profilaxis antibiótica (PA) el 60\% tuvo un conocimiento de nivel medio $23.3 \%$ bajo y $16.7 \%$ alto. En la dimensión sistemáticamente comprometidos (SC) el 70\% tuvieron un conocimiento de nivel bajo, el 23.3\% medio y el $6.7 \%$ alto. Concluyendo que los cirujanos dentistas de Chachapoyas están en un nivel de conocimiento medio sobre prescripción antibiótica racional.
\end{abstract}

Palabras clave: prescripción, antibiótica, resistencia antibiótica

\begin{abstract}
The objective of the research was to determine the level of knowledge about rational antibiotic prescription of dental surgeons in the city of Chachapoyas in 2020 . The present study was quantitative. The sample was constituted by 30 Dental surgeons who cooperated with the investigation. For data recording, the survey technique was applied and the knoledge questionnaire as an instrument, the dat were analyzed with descriptive frequency statistics. The reslts showed that of $100 \%$ of those surveyed, $70 \%$ had a médium level knowledge, 16.7\% low and 13.3\% high. In the dimensiono first (PET) 53\% hat a médium level knowledge, 36.7\% low and 10\% high. In the antibiotic dimensión of choice in allergic patientes (AEPA), 63.4\% had a low level knowledge, $23.3 \%$ low and only $13.3 \%$ high. In the antibiotic prophylaxis (PA) dimensión, 60\% had a médium level knowledge, $23.3 \%$ low an 16.7\% high. In the dimensión systematically that Chachapoyas Dental Surgeons are at a médium level of knowledge about rational antibiotic prescription.
\end{abstract}

Keywords: prescription, antibiotic, antibiotic resistance

\footnotetext{
Tesista en Estomatología, Universidad Nacional Toribio Rodríguez de Mendoza. Email: 7332441932@untrm.edu.pe ${ }^{2}$ Asesor: Docente asociado a tiempo completo en la facultad de Ciencias de la Salud, Universidad Nacional Toribio Rodríguez de Mendoza. Correo electrónico: franz.coronel@untrm.edu.pe
} 


\section{INTRODUCCIÓN}

La salud oral amerita desde luego una atención exclusiva de parte de los profesionales de odontología, para que la sociedad goce de una buena salud bucal general, sin embargo, desde la existencia de la humanidad la condición de la salud bucal se ha visto seriamente vulnerable mostrándose altos niveles de prevalencia de enfermedades odontoestomatológicas.

Asimismo, Batellino L y Col (1993) menciona que "la utilidad de los antibióticos desde su descubrimiento ha significado el control y tratamiento de patologías infecciones incluidas las de aparición en la cavidad oral" (p. 12).

Los antibióticos, por su acción frente a las diferentes patologías, son utilizadas como: la primera opción terapéutica farmacológica, como las diferentes enfermedades infecciosas de origen bacteriano, el origen de los antibióticos está situado en sustancias naturales, semisintéticas o sintéticas los cuales tienen su mecanismo de acción inhibiendo los microorganismos, interviniendo a nivel molecular, ya sea metabólicamente o en estructuras específicas del microorganismo. (Bernabé M, Flores D y Martínez M, 2015,p.5).

Por otro lado, en la práctica odontológica: la prescripción de antibióticos, está sustentada en el origen de la enfermedad, de los cuales la gran mayoría de enfermedades son de origen bacteriano. La prescripción de antibióticos en odontología, la prescripción de antibióticos en odontología, también está estrechamente relacionado a combatir infecciones orales de origen odontogénico, ya sean en infecciones focales y locales, o en la dimensión a tejidos y órganos vecinos. Al utilizar un medicamento para tratar problemas de salud de un paciente, se tiene que tener en cuenta aspectos del fármaco como: características básicas de eficacia, seguridad y accesibilidad, por lo que, los antibióticos desempeñan desde luego una acción importante en la prevención y tratamiento de enfermedades, también como paliativo de los sintomatologías, es así, que el empleo optimo y racional de estos antibióticos será primordial para la correcta práctica profesional y se verá reflejado en la mayoría del estado de salud del paciente. (Baos V, 2000, p. 3-5).

En la actualidad, la prescripción antibiótica está basada en la experiencia de la práctica del operador, ignorando muchas veces el uso de protocolos establecidos, todo ello se ve reflejado en el incremento de resistencia bacteriana a los fármacos por uso de antibióticos de manera imprudente y sin control, por lo que algunos microorganismos que no suponían un riesgo para la salud de su tratamiento" (Esparza L, 2016, p. 34).

Según Gonzáles A y Guzmán J. (2012), en odontología también se ha incrementado la prescrip- ción irracional de antibióticos por lo que constantemente se trata de encontrar las medidas necesarias para que ayuden a mitigar está problemática a través de publicaciones de investigaciones.

La inadecuada prescripción farmacológica que tiene como causa principal, la falta de capacitación constante, por parte de los Cirujanos Dentistas en el área de farmacología. Entonces, el uso incorrecto de antibiótico en la práctica clínica, ya sea particular o pública, es una de las principales razones para que aumente los riesgos de resistencia bacteriana en la población.

En la región de Amazonas existe un gran números de Cirujanos Dentistas que ejercen tanto en la práctica pública como privada, interviniendo diferentes patologías odontológicas que ameritan la prescripción farmacológica para su tratamiento, por lo que nace la necesidad de conocer cuál es nivel de conocimiento sobre prescripción antibiótica racional de los Cirujanos Dentistas, el objetivo de la presente investigación fue determinar el nivel de conocimiento sobre sobre prescripción antibiótica racional de los Cirujanos Dentistas, en la ciudad de Chachapoyas en el año 2020.

\section{MATERIAL Y MÉTODO}

El estudio, fue de enfoque cuantitativo, nivel descriptivo, de tipo: observacional, prospectivo, transversal y univariado. Además, el estudio final fue procesado en los programas Microsoft Word y Excel, seguido del paquete estadístico de software SPSS-25. Los resultados finales se aplicaron en tablas simples y figuras de barras. (Supo,2016, p.2-19).

La población de estudio estuvo conformada por 30 Cirujanos Dentistas de Chachapoyas. durante el método de investigación se utilizó el método Inductivo-Deductivo. (Bunge, 2014, p. 225).

Se utilizó un cuestionario como instrumento de recolección de datos. 


\section{RESULTADOS}

Figura 1

Nivel de conocimiento sobre prescripción antibiótica racional de los Cirujanos Dentistas, Chachapoyas, 2020

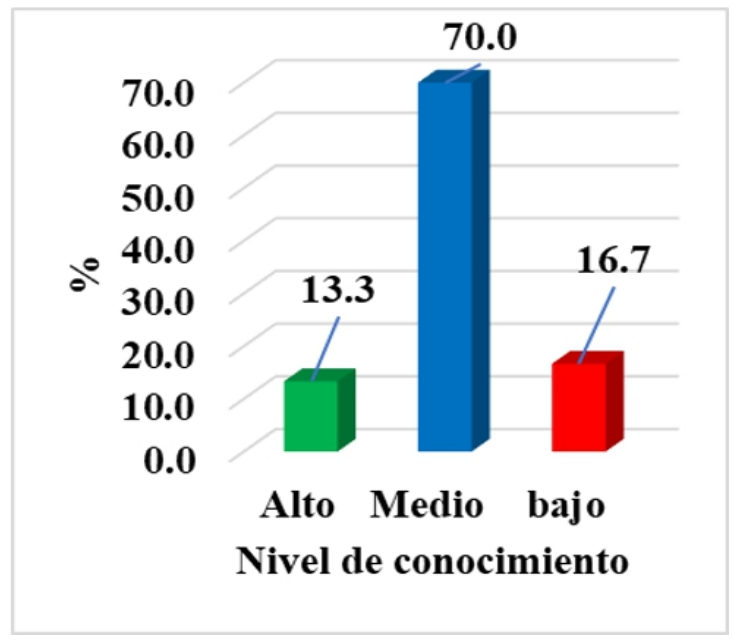

En la Figura 1, se determinó que, del $100 \%$ de la población de los Cirujanos Dentistas, el 70\% (21) tienen un conocimiento de nivel medio sobre prescripción antibiótica racional, mientras que el $16.7 \%$ (5) bajo y el 13.3\% (4) alto.

\section{Figura 2}

Nivel de conocimiento sobre prescripción antibiótica racional en función a sus dimensiones de los Cirujanos Dentistas, Chachapoyas, 2020

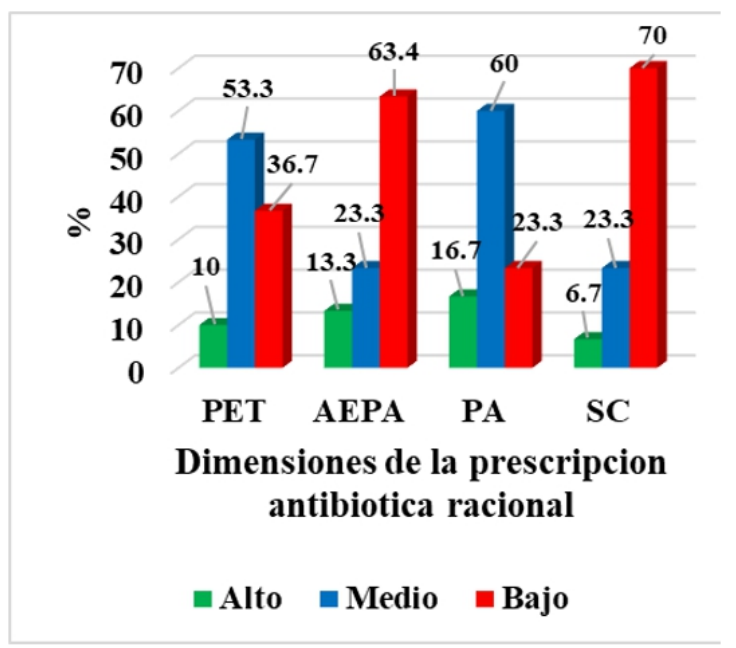

Leyenda:

PET: Primera elección para el tratamiento de infecciones odontogénicas.

AEPA: Antibiótico de elección en pacientes alérgicos.

PA: Profilaxis antibiótica.

SC: Sistemáticamente comprometidos.
En la figura 2, se realizó una descripción del nivel de conocimiento de las dimensiones sobre la prescripción antibiótica racional:

En la dimensión primera elección en pacientes alérgicos (PET) se observa que del $100 \%$ de los Cirujanos Dentistas el 53.3\% (16) tienen un conocimiento de nivel medio, el 36.7 (11) bajo y solo el 10\% (3) alto.

En la dimensión de antibiótico de elección en pacientes alérgicos (AEPA) se observa que del 100\% de los Cirujanos Dentistas el 63.4\% (19) tienen un conocimiento de nivel bajo, el $23.3 \%$ medio y solo el 13.3 (4) alto.

En la dimensión Profilaxis antibiótica (PA) se observa que del $100 \%$ de los Cirujanos Dentistas el $60 \%$ (21) tienen un conocimiento de nivel medio, el $23.3 \%$ (7) bajo y solo el $16.7 \%$ (7) bajo y solo el $16.7 \%$ (5) alto.

En la dimensión sistemáticamente comprometidos (SC) se observa que del $100 \%$ de los Cirujanos Dentistas el 70\% (21) tiene un conocimiento de nivel bajo, el $23.3 \%$ (7) medio y solo el $6.7 \%$ (2) alto.

\section{DISCUSIÓN}

En la figura 1, nivel de conocimiento sobre prescripción antibiótica racional de los Cirujanos Dentistas, Chachapoyas, 2020. Los resultados reflejan que el $70 \%$ está en un nivel medio, el 16.7\% en un nivel bajo y el $13.3 \%$ en un nivel alto. Comparando los resultados de la presente investigación con los estudios realizados por Cerrón, $M$ (2018). Sus resultados fueron que $45 \%$ tuvieron un conocimiento de nivel regular. Estos resultados reflejan una similitud con los estudios realizados del presente estudio. Asimismo, los resultados realizados por Ramírez, E. (2016). 63\% tuvo un conocimiento de nivel regular, reflejan similitud con los estudios realizados por cerrón y con los resultados de la presente investigación. Estos resultados indican que la mayoría de los odontólogos conocen parcialmente sobre prescripción antibiótica ya que obtuvieron un nivel de conocimiento medio.

Mientras que, en los estudios realizados por Contreras, M. (2015). Sus realizados indicaron que $63 \%$ tuvo un conocimiento de nivel malo. Difiriendo totalmente con los resultados obtenidos por Ramírez, Cerrón y con los del presente estudio. Está diferencia puede ser por los diferentes escenarios donde se realizaron las investigaciones.

La figura 2, mostró el nivel de conocimiento sobre prescripción antibiótica en sus dimensiones:

En la dimensión de primera elección para el tratamiento de infecciones (PET), se determinó que el $53.3 \%$ tuvo un conocimiento medio. Sin embargo, afrontando con los resultados obtenidos por Ramírez, 
E. (2016). En el que reflejó un 48\% de nivel de conocimiento malo. No concordando con los resultados del presente estudio. Revisando la literatura se encontró estudios realizados por Cedillo, M. y Delgado, D. (2018). Denotó 61.6\% obtuvieron un nivel de conocimiento bueno. Difiriendo con los resultados obtenidos por Ramírez y del presente estudio.

Respecto a la interpretación en la dimensión de antibiótico de elección en pacientes alérgicos (AEPA) para el tratamiento de infecciones odontogénicos al de primera elección, se obtuvo que el $63.4 \%$ tuvo un nivel de conocimiento bajo. Comparando con los resultados por Ramírez, E. (2016). Que arrojaron un $42 \%$ de nivel de conocimiento malo. Existiendo una similitud entre ambos estudios, esto quiere decir que los Cirujanos Dentistas no tienen conocimiento respectivo sobre dicha dimensión.

Por otro lado, en la dimensión de profilaxis antibiótica (PA). Loa resultados reflejan que el 60\% tienen un conocimiento de nivel medio. Comparando los resultados obtenidos en esta misma dimensión por Cedillo, M. y Delgado,

D. (2018). Sus resultados indican que $60.5 \%$ tuvieron un nivel de conocimiento bueno; difiriendo totalmente con los resultados del presente estudio. Está diferencia puede ser porque las investigaciones se realizaron en diferentes países, considerando que los odontólogos estudiados por Cedillo y Delgado tienen mayor conocimiento sobre la prescripción antibiótica en esta dimensión.

Asimismo, en la dimensión en pacientes sistemáticamente comprometidos del total de los encuestados, el 70\% tienen un nivel de conocimiento bajo. Sin embargo, en los estudios realizados por Uyeki, P. (2016). Demostró que el 40\% tuvieron un nivel de conocimiento bueno, difiriendo con los resultados del presente estudio. Ante esta diferencia se puede concluir que los odontólogos investigados por Uyeki están mejor preparados que los de la presente investigación.

\section{CONCLUSIONES}

Los Cirujanos Dentistas de la ciudad de Chachapoyas tienen un nivel de conocimiento medio sobre prescripción antibiótica racional.

En la dimensión de primera elección para el tratamiento de infecciones odontogénicas, los Cirujanos Dentistas tienen un nivel de conocimiento medio.

Al evaluar en la dimensión de antibiótico de elección en pacientes alérgicos, los Cirujanos Dentistas tuvieron un nivel de conocimiento bajo.
Por otro lado, al evaluar la dimensión sobre profilaxis antibiótica, el nivel de conocimiento de los Cirujanos Dentistas fue medio.

En la dimensión de pacientes sistemáticamente comprometidos, los Cirujanos Dentistas obtuvieron un nivel de conocimiento bajo.

\section{REFERENCIAS BIBLIOGRÁFICAS}

Aguirre, P. (2013). Nivel de conocimiento sobre profilaxis antibiótica de endocarditis infecciosa previa a procedimientos odonto lógicos en internos de odontología de tres universidades de Lima-2015. (tesis pregra do).https://hdl.handle.net/20.500.12672/35

Batellino, L y Col. (2013). Conocimiento farmacológico, conducta de prescripción y propuesta para mejorar el empleo de medicamentos en odontólogos. Revista de salud pública, 27 (4).

Carranza, G. (2017). Conocimiento del uso de antibióticos por el gremio odontológico egresado de la facultad de odontología. Guatemala: Revista odontológica.

Cedillo, M. Delgado, D. (2018). Conocimientos de los odontólogos del área urbanos de cuenca sobre los antibióticos. (tesis pregrado).

Cerrón, M. (2018). Nivel de conocimiento sobre prescripción antibióticos en infecciones odontogénicas en internos de odontología de la universidad nacional Federico Villarreal, 2018. (tesis pregrado).

Contreras, M. (2015). Nivel de conocimiento sobre prescripción antibiótica racional de los cirujanos dentistas de la ciudad de Puno Agosto-septiembre del 2015. (tesis pregrado).

Curay, S. (2015). Nivel de conocimiento sobre prescripción antibiótica racional de los cirujanos dentistas docentes de la universidad señor de Sipán Lambayeque-Perú, 2015. (tesis pregrado).

Gallegos, S. (2015). Preferencia de prescripción antibiótica ante infecciones odontológicas por estomatólogos. Revista de ciencias médicas la Habana, 2520-9078(30). 
Pérez, r. (2017). Nivel de conocimiento de los cirujanos dentistas sobre prescripción racional antimicrobianos en odontología del distrito de Chiclayo, Lambayeque 2017. (tesis pregrado).

Ramírez, E. (2016). Nivel de conocimiento sobre prescripción antibiótica racional de los cirujanos dentistas del distrito de Chimbote , provincia de Santa-Áncachs 2016. (tesis pregrado).

Supo, J. (2016). Metodología de investigación cientifica de las bases conceptuales de la investigación. Arequipa. Edit. Universitaria. Yanahuara. Arequipa.

Uyeki, P. (2016). Nivel de conocimiento sobre prescripción antibiótica racional de los cirujanos dentistas docentes de la clínica odontológica ULADECH católica, Chimbote, 2016. (tesis pregrado). 